\title{
Numerical simulations of Hall MHD small-scale dynamos
}

\author{
Daniel O. Gómez ${ }^{1,2}$ Pablo D. Mininni ${ }^{1,3}$ \\ and Pablo Dmitruk ${ }^{1}$ \\ ${ }^{1}$ Departamento de Física, FCEN-UBA, (1428) Buenos Aires, Argentina \\ ${ }^{2}$ Instituto de Astronomía y Física del Espacio, CC 67 Suc 28, (1428) Buenos Aires, Argentina \\ ${ }^{3}$ National Center for Atmospheric Research, Boulder, CO 80307, USA
}

\begin{abstract}
Much of the progress in our understanding of dynamo mechanisms, has been made within the theoretical framework of magnetohydrodynamics (MHD). However, for sufficiently diffuse media, the Hall effect eventually becomes non-negligible. We present results from three dimensional simulations of the Hall-MHD equations subjected to random non-helical forcing. We study the role of the Hall effect in the dynamo efficiency for different values of the Hall parameter, using a pseudospectral code to achieve exponentially fast convergence.
\end{abstract}

Keywords. ISM, magnetic fields, MHD, turbulence.

\section{Introduction}

The generation of magnetic fields by dynamo activity plays a very important role in a wide range of astrophysical objects, ranging from stars to clusters of galaxies. The gas in these objects is characterized by turbulent flows. Helical flows have proved efficient in generating large-scale dynamos, i.e. on scales larger than the energy-containing eddies of the flow. On the other hand, non-helical flows can be instrumental in generating small-scale dynamos (see Kazantsev (1968), also Haugen et al. (2004)). For sufficiently diffuse media such as the one that pervades the ISM, the Hall current eventually becomes non-negligible and its effect needs to be considered. We present results from three dimensional simulations of the Hall-MHD equations subjected to random non-helical forcing. We study the role of the Hall effect in the dynamo efficiency for different values of the Hall parameter.

\section{Hall-MHD equations}

Incompressible Hall-MHD is described by the modified induction equation and the equation of motion, i.e. dissipative Navier-Stokes equation,

$$
\begin{aligned}
\frac{\partial \boldsymbol{B}}{\partial t} & =\nabla \times[(\mathbf{U}-\epsilon \nabla \times \boldsymbol{B}) \times \mathbf{B}]+\eta \nabla^{2} \mathbf{B} \\
\frac{\partial \mathbf{U}}{\partial t} & =-(\mathbf{U} \cdot \nabla) \mathbf{U}+(\boldsymbol{B} \cdot \nabla) \boldsymbol{B}-\nabla\left(P+\frac{B^{2}}{2}\right)+\mathbf{F}+\nu \nabla^{2} \mathbf{U}
\end{aligned}
$$

where $\nabla \cdot \mathbf{B}=0=\nabla \cdot \mathbf{U}$ and $\mathbf{F}$ denotes a solenoidal and non-helical external force, deltacorrelated in time. The velocity $\mathbf{U}$ and the magnetic field $\mathbf{B}$ are expressed in units of a characteristic speed $U_{0}, \epsilon$ measures the relative strength of the Hall effect, $\eta$ is the magnetic diffusivity, and $\nu$ is the kinematic viscosity. Note that the measure of Hall effect $\epsilon$ can be written as $\epsilon=\frac{c}{\omega_{p i} L_{0}} \frac{U_{A}}{U_{0}}$ where $L_{0}$ is a characteristic length scale (the size of the box is $2 \pi L_{0}$ ).

\section{Results}

We performed simulations of the HMHD equations with $256^{3}$ spatial resolution, using a pseudospectral code - Mininni et al. (2005). We first generate a stationary hydrodynamic turbulence by applying a non-helical forcing. In a second stage, a random and small magnetic field is 


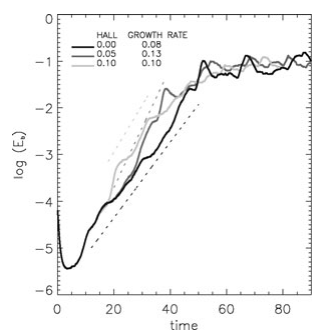

Figure 1. (a) Kinetic (thick) and magnetic (thin) dissipation rates vs. time for $\epsilon=0.00,0.05,0.10$. (b) Magnetic energy vs. time, listing the corresponding growth rates.
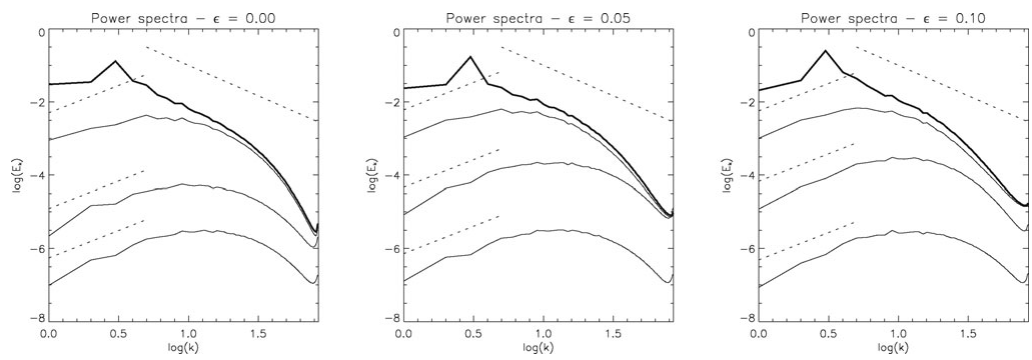

Figure 2. Total energy spectrum (thick trace) at $t=72$. Magnetic energy spectra at $t=18,36,72$ (from left to right) are also shown. The Kolmogorov and Kazantsev spectra are overlaid (dotted trace) for reference.

introduced at small scales. The viscous (thick trace) and resistive (thin) dissipation rates vs. time are shown in Fig. 1a for three runs with different values of the Hall parameter $\epsilon$. The exponentially fast growth of magnetic energy is displayed in Fig. 1b. Even though the growth rate for moderate Hall $(\epsilon=0.05)$ is larger than from a purely MHD run (i.e. $\epsilon=0.00)$, for somewhat larger Hall $(\epsilon=0.10)$ the growth rate is reduced, confirming similar results by Mininni et al. (2005) for large-scale dynamos. The growth of the magnetic energy spectrum is shown in Fig. 2. The Kazantsev slope $E_{k} \propto k^{3 / 2}$ (Kazantsev (1968)) provides a reasonable approximation at small wavenumbers for all these cases, while the kinetic energy spectrum remains always close to Kolmogorov (i.e. $E_{k} \propto k^{-5 / 3}$ ). At saturation, the total magnetic energy reaches a sizeable fraction of the total kinetic energy ( $15 \%$ to $20 \%$ ).

The key to understand the impact of the Hall effect on the dynamo is to study its role on the shell-to-shell energy transfer, which is discussed in Mininni et al. (2009) (see details in Mininni, Alexakis \& Pouquet (2007)).

\section{References}

Mininni, P. D., Alexakis, A., \& Pouquet, A. 2007, J. Plasma Phys. 73, 377

Haugen, N. E. L., Brandenburg, A., \& Dobler, W. 2004, Phys. Rev. E 70, 016308

Mininni, P. D., Gómez, D. O., \& Mahajan, S. M. 2005, Ap.J. 619, 1019

Kazantsev, A. P. 1968, Sov. Phys. JETP 26, 1031

Mininni, P. D., Dmitruk, P., \& Gómez, D. O. 2009, in prep. 